\title{
Identification of blue high proper motion objects in the Tycho-2 and 2MASS catalogues using Virtual Observatory tools
}

\author{
F. M. Jiménez-Esteban ${ }^{1,2,3}$, J. A. Caballero ${ }^{4}$, and E. Solano ${ }^{1,2}$ \\ ${ }^{1}$ Centro de Astrobiología (INTA-CSIC), Departamento de Astrofísica, PO Box 78, 28691 Villanueva de la Cañada, Madrid, Spain \\ e-mail: fran.jimenez-esteban@cab.inta-csic.es \\ 2 Spanish Virtual Observatory, Spain \\ 3 Saint Louis University, Madrid Campus, Division of Science and Engineering, Avenida del Valle 34, 28003 Madrid, Spain \\ ${ }^{4}$ Centro de Astrobiología (INTA-CSIC), Departamento de Astrofísica, Carretera de Ajalvir km 4, 28850 Torrejón de Ardoz, Spain
}

Received 16 June 2010 / Accepted 12 September 2010

\section{ABSTRACT}

\begin{abstract}
Aims. With available Virtual Observatory tools, we looked for new bright blue high proper motion objects in the entire sky: white dwarfs, hot subdwarfs, runaway OB stars, and early-type stars in nearby young moving groups.

Methods. We performed an all-sky cross-match between the optical Tycho-2 and near-infrared 2MASS catalogues with Aladin, and

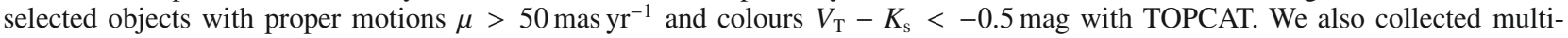
wavelength photometry, constructed the spectral energy distributions and estimated effective temperatures from fits to atmospheric models with VOSA for the most interesting targets.

Results. We assembled a sample of 32 bright blue high proper motion objects, including ten sdO/B subdwarfs, nine DA white dwarfs, five young early-type stars (two of which are runaway stars), two blue horizontal branch stars, one star with poor information, and five objects reported for the first time in this work. These last five objects have magnitudes $B_{\mathrm{T}} \approx 11.0-11.6$ mag, effective temperatures $T_{\text {eff }} \approx 24000-30000 \mathrm{~K}$, and are located in the region of known white dwarfs and hot subdwarfs in a reduced proper motion-colour diagram. We confirmed the hot subdwarf nature of one of the new objects, Albus 5, with public far-ultraviolet spectroscopic data obtained with FUSE.
\end{abstract}

Key words. astronomical databases: miscellaneous - virtual observatory tools - stars: early-type - stars: peculiar - subdwarfs - white dwarfs

\section{Introduction}

Bright objects with blue colours and high proper motions are rare in the sky. By "bright" we mean sources with optical magnitudes in the range covered by classical astronomical catalogues, such as the Bonner Durchmusterung (Schonfeld 1886; Argelander 1903), Córdoba Durchmusterung (Thome 1894), Henry Draper (Cannon \& Pickering 1925, and previous versions), or the most recent Hipparcos (Perryman et al. 1997) and Tycho-2 (Høg et al. 2000) catalogues. These catalogues typically have higher limit magnitudes between 9 and $11 \mathrm{mag}$ in the visible. Blue colours are indicative of high effective temperatures, while high proper motions of tens of mas $\mathrm{yr}^{-1}$ imply short heliocentric distances or large tangential velocities at moderate distances.

There are few possible kinds of bright blue high proper motion objects. They can be nearby white dwarfs, hot subdwarfs, runaway OB stars, or early-type stars in nearby young moving groups. Most of the known blue objects (e.g., massive early-type stars in massive clusters) in the Galaxy are located far away and, therefore, have low proper motions and are apparently faint save for a few exceptions. Likewise, a wealth of objects with high proper motions are known, but the bulk of them are neighbouring late-type stars with red colours. Blue extragalactic objects are also known, but they present a negligible proper motion.

Bright blue high proper motion objects are important for many fields in astrophysics. Bright white dwarfs and hot subdwarfs, for instance, are extensively used as spectrophotometric standard stars (Stone 1977; Oke \& Gunn 1983; Oke 1990), class prototypes, or as tracers of the Population II in the Galaxy. Their apparent high optical brightnesses facilitate the determination of physical parameters at extreme conditions (very high temperatures and gravities) in a relatively undemanding way. Similarly, runaway OB stars and early-type stars in young moving groups are fundamental for understanding the evolution and evaporation of star-forming regions.

In this paper, we used Tycho-2 and the Two Micron All Sky Survey Point Source (2MASS; Skrutskie et al. 2006) catalogues and Virtual Observatory tools to look for the bluest objects $\left(V_{\mathrm{T}}-K_{\mathrm{s}} \leq-0.5 \mathrm{mag}\right)$ with the highest proper motions

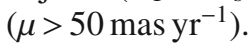

\section{Analysis and results}

\subsection{Tycho-2/2MASS cross-match}

On the one hand, Tycho-2 gives position and proper motion information for the 2.5 million brightest stars in the sky. Photometric data for two pass-bands $\left(B_{\mathrm{T}}\right.$ and $V_{\mathrm{T}}$, close to Johnson B and V; Perryman et al. 1997) are also provided.

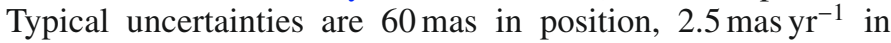
proper motion, and $0.1 \mathrm{mag}$ in photometry. On the other hand, 2MASS contains photometric data for 471 million sources in the $J, H$, and $K_{\mathrm{s}}$ near-infrared bands. Typical photometric and astrometric uncertainties of 2MASS are less than 0.03 mag and 100 mas, respectively. 
In this work we took advantage of Virtual Observatory ${ }^{1}$ (VO) tools to cross-match the whole Tycho-2 and 2MASS catalogues. The VO is a project designed to provide the astronomical community with the data access and the research tools necessary to enable the exploration of the digital, multi-wavelength universe resident in the astronomical data archives. In particular, we made use of the scripting capability of Aladin ${ }^{2}$ (Bonnarel et al. 2000) to perform our cross-match, a VO-compliant software that allows users to visualise and analyse digitised astronomical images, and superimpose entries from astronomical catalogues or databases available from the VO services. To avoid memory overflow during data processing, we divided the full sky sphere into overlapping circular regions of $4.5 \mathrm{deg}^{2}$. The useful and powerful Aladin script mode allowed us to readily perform the following workflow for each region:

- Select all Tycho-2 sources with proper motion $\mu>$

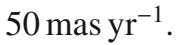

- Select all 2MASS sources within a radius of $40 \operatorname{arcsec}$ around each selected high proper motion Tycho-2 source.

- Convert 2MASS coordinates into J2000.0 epoch using the proper motion of the related Tycho-2 source.

- Select for each Tycho-2 source the nearest proper motioncorrected 2MASS source.

In the second step, we used such a large radius to account for the expected high proper motion of the objects along the years. We took as reference the maximum proper motion known so far (that of the Barnard star, $10.3 \operatorname{arcsec} \mathrm{yr}^{-1}$ ) and the maximum possible temporal separation between Tycho-2 and 2MASS observations $(\approx 4 \mathrm{yr})$.

During the cross-match process, we imposed a good quality flag $\left(\mathrm{Qflg}=\mathrm{A}, \mathrm{B}, \mathrm{C}\right.$, or D) in $K_{\mathrm{S}}$ and an error lower than $0.3 \mathrm{mag}$ in $V_{\mathrm{T}}$. It returned more than 300000 identifications. However, because we worked with overlapping regions, an important fraction of the identifications were duplicated. We used TOPCAT ${ }^{3}$ to purge the duplicated data and to carry out the subsequent analyses. TOPCAT is an interactive graphical viewer and editor for tabular data that allows the user to examine, analyse, combine, and edit astronomical tables. After purging the data we ended with a sample of 157184 sources fulfilling all the above criteria.

In Fig. 1 we show the histograms of angular separations $\rho$ between the cross-matched Tycho-2 and 2MASS proper motion-corrected sources, in both directions, right ascension and declination. Most of the separations (98.3\%) are lower than 0.50 arcsec. Quantitatively, the mean angular separation and standard deviation are $\overline{\rho_{\alpha}}=0.00 \operatorname{arcsec}$ and $\sigma_{\rho_{\alpha}}=0.23 \operatorname{arcsec}$ for the right ascension, and $\overline{\rho_{\delta}}=0.01 \operatorname{arcsec}$ and $\sigma_{\rho_{\delta}}=$ 0.26 arcsec for the declination. Thus, we discarded 1800 (spurious) matches with separations higher than $3 \sigma_{\rho}$ in any of the spatial directions, which reduced the total number of sources to 155384.

\subsection{Colour selection}

We show the reduced proper motion-colour diagram of the cross-matched sources in Fig. 2. The reduced proper motion, $H_{V_{\mathrm{T}}}$, is defined as $H_{V_{\mathrm{T}}}=V_{\mathrm{T}}+5 \log \mu+5$, where $\mu$ is the proper motion in arcseconds per year. It can also be expressed as $H_{V_{\mathrm{T}}}=M_{V_{\mathrm{T}}}+5 \log v_{\mathrm{t}}-3.3$, where $M_{V_{\mathrm{T}}}$ is the $V_{\mathrm{T}}$-band absolute magnitude and $v_{\mathrm{t}}$ is the transverse velocity in kilometers per

\footnotetext{
1 http://www.ivoa.net

2 http://aladin.u-strasbg.fr/

${ }^{3}$ http://www.star.bris.ac.uk/ mbt/topcat/
}

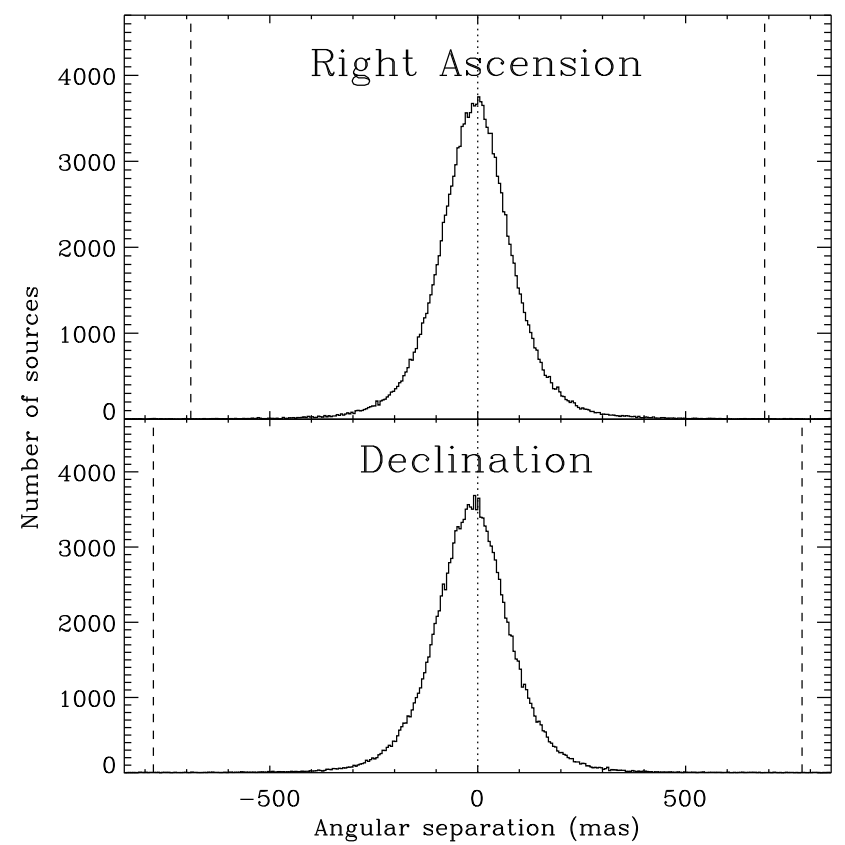

Fig. 1. Histograms of the angular separation in right ascension (upper panel) and declination (lower panel) between the Tycho-2 and the closest 2MASS counterpart, after correcting the 2MASS coordinates for Tycho- 2 proper motion. The vertical dashed lines indicate the maximum separation allowed for a reliable cross-match, $3 \sigma_{\rho_{\alpha}}=0.69 \operatorname{arcsec}$ in right ascension, and $3 \sigma_{\rho_{\delta}}=0.78$ arcsec in declination.

second. The $V_{\mathrm{T}}-K_{\mathrm{s}}$ colour is a suitable effective-temperature indicator in field objects. The reduced proper motion diagram therefore constitutes a powerful tool for segregating members of kinematically-distinct stellar populations (e.g. Lépine \& Shara 2005). In particular, it is possible to distinguish three types of objects from Fig. 2: Population I (upper branch), Population II stars (middle branch) and white dwarfs (very tiny lower branch).

For selecting blue high proper motion candidates, we applied a very simple selection criterion: $V_{\mathrm{T}}-K_{\mathrm{S}}<-0.5 \mathrm{mag}$, depicted in Fig. 2 with a vertical dashed line. In total, 33 objects showed bluer colour than this value. This simple selection criterion produced an appropriate number of sources that could be studied in detail in a reasonable time scale.

\subsection{Visual inspection, object rejection, and final sample}

We visually inspected the 33 selected blue high proper motion candidates with Aladin. We compared and blinked two sets of images separated several decades in time, optical DSS-1 and near-infrared 2MASS, to easily confirm the high proper motion of our candidates. The Tycho-2 sources and 2MASS counterparts were superimposed on the images to assess the reliability of the cross-match. Besides, we used other available astro-photometric databases, such as the Astrographic Catalogue AC2000.2 (Urban et al. 1998), USNO-B1 (Monet et al. 2003), SuperCOSMOS Sky Survey (Hambly et al. 2001), DENIS (Epchtein et al. 1997) and the Positions and Proper MotionsExtended PPMX (Röser et al. 2008), to verify the high proper motion (Fig. 3).

Of the 33 blue high proper motion candidates, we discarded TYC 8250-687-1, for which Tycho-2 provided an erroneous proper motion after mixing the star 2MASS 13052225-4740536 ( $V<10 \mathrm{mag}$ ) with the close $(\rho \approx 14 \mathrm{arcmin})$ bright extended 
Table 1. Already known bright blue high proper motion objects.

\begin{tabular}{|c|c|c|c|c|c|c|}
\hline TYC & $\begin{array}{c}\alpha^{a} \\
(\mathrm{~J} 2000)\end{array}$ & $\begin{array}{c}\delta^{a} \\
(\mathrm{~J} 2000)\end{array}$ & $\begin{array}{c}\pi^{b} \\
(\mathrm{mas})\end{array}$ & Name & Object type & Ref. \\
\hline 6429-796-1 & 014348.55 & -240510.2 & $3 \pm 4$ & CD-24 731 & $\mathrm{sdO} / \mathrm{B}$ hot subdwarf in a close binary system & $1,2,3$ \\
\hline $8055-1270-1$ & 021630.58 & -513043.8 & $21.22 \pm 0.12$ & $\phi$ Eri & Young B8V star in Tucana-Horologium & 4 \\
\hline $9145-601-1$ & 031031.02 & -683603.4 & $97.7 \pm 1.8$ & WD 0310-688 & DA3.1 white dwarf in a wide binary system & $5,6,7$ \\
\hline $83-1181-1$ & 044442.13 & +003405.2 & $3.0 \pm 0.6$ & HD 30112 & Runaway B2.5V star & 8 \\
\hline $3734-2324-1$ & 050530.61 & +524951.9 & $18 \pm 3$ & WD $0501+527$ & DA1 white dwarf & $9,10,6$ \\
\hline $9166-716-1$ & 053140.35 & -695302.2 & $\ldots$ & AA Dor & sdO/B hot subdwarf in an eclipsing binary system & $11,2,12$ \\
\hline $7075-2143-1$ & 060420.27 & -321020.8 & $3.2 \pm 0.3$ & $72 \mathrm{Col} \mathrm{AB}$ & Runaway B2.5V star in a binary system & $13,14,8$ \\
\hline 7613-1087-1 & 062312.20 & -374129.0 & $\ldots$ & WD 0621-376 & DA1 white dwarf & $15,10,6$ \\
\hline 7664-1961-1 & 081031.60 & -403310.0 & $\ldots$ & CD-40 3927 & sdO hot subdwarf & 2 \\
\hline 4547-1009-1 & 092119.18 & +814327.6 & $4 \pm 2$ & AG+81 266 & sdO hot subdwarf & 16,2 \\
\hline $3429-1180-1$ & 093046.78 & +481623.8 & $6.2 \pm 1.8$ & BD+48 1777 & sdOp hot subdwarf & $17,18,2$ \\
\hline $3005-579-1$ & 103516.71 & +402113.6 & & PB 385 & sdB hot subdwarf & 19,2 \\
\hline 1984-97-1 & 113705.10 & +294758.3 & $63 \pm 4$ & WD $1134+300$ & DA2.5 white dwarf & $20,6,7$ \\
\hline $2541-4-1$ & 132335.26 & +360759.5 & $\ldots$ & NSV 19768 & sdOp hot subdwarf & $21,22,2$ \\
\hline $5545-1390-1$ & 133013.64 & -083429.5 & $58 \pm 4$ & WD 1327-083 & DA3.7 white dwarf in a wide binary system & $23,6,7$ \\
\hline 2004-242-1 & 133824.76 & +292156.0 & $5.5 \pm 1.5$ & BD+30 2431 & B3Vp halo blue horizontal branch star & $24,25,26$ \\
\hline $7832-2490-1$ & 145831.93 & -430802.3 & $8.52 \pm 0.18$ & $\beta$ Lup & Young B2III star in Sco OB2 & 27,28 \\
\hline 7839-874-1 & 152607.13 & -392919.2 & & CD-39 9849 & $\mathrm{sdO} / \mathrm{B}$ hot subdwarf ${ }^{c}$ & 29,30 \\
\hline $7853-559-1$ & 162333.84 & -391346.2 & $76 \pm 3$ & WD 1620-391 & DA2 white dwarf in a wide binary system & $31,6,7$ \\
\hline $3885-860-1$ & 164825.64 & +590322.7 & $94 \pm 3$ & DN Dra & DAV4 white dwarf, pulsating & $32,10,6$ \\
\hline $8359-3650-1$ & 182658.42 & -455806.5 & $11.74 \pm 0.17$ & $\alpha$ Tel & Young B3IV star in Sco OB2 & 33,28 \\
\hline $3531-1327-1$ & 183408.90 & +482717.0 & & $\mathrm{BD}+482721$ & sdB hot subdwarf & $34,2,35$ \\
\hline 2161-1038-1 & 203421.88 & +250349.7 & $64 \pm 3$ & WD $2032+248$ & DA2.5 white dwarf & $23,6,7$ \\
\hline 8424-1008-1 & 213910.61 & -460551.5 & $3.8 \pm 1.7$ & HD 205805 & sdB hot subdwarf, helium-poor & 36,2 \\
\hline 2211-1613-1 & 215941.97 & +262557.4 & $9.1 \pm 1.2$ & IS Peg & sdO hot subdwarf in a binary system & 37,2 \\
\hline $8441-1261-1$ & 221411.91 & -491927.3 & $\ldots$ & WD 2211-495 & DA1 white dwarf & $15,10,6$ \\
\hline $6401-39-1$ & 231230.63 & -210643.4 & $0.0 \pm 1.4$ & HD 218970 & B2-3IV/Vp star found far from the galactic plane & $18,38,39$ \\
\hline
\end{tabular}

Notes. ${ }^{(a)}$ Equatorial coordinates from Tycho-2. ${ }^{(b)}$ Trigonometric parallax from Hipparcos. ${ }^{(c)}$ CD-39 9849 was classified as a blue horizontal branch star or sdO/B hot subdwarf by Drilling \& Bergeron (1995). We classify it as an sdO/B hot subdwarf based on its location in the reduced proper motion-colour diagram.

References. (1) Haro \& Luyten (1962); (2) Kilkenny et al. (1988); (3) O’Toole \& Heber (2006); (4) Zuckerman et al. (2001); (5) Thackeray (1961); (6) Caballero \& Solano (2007); (7) Sion et al. (2009); (8) Hoogerwerf et al. (2001); (9) Greenstein (1969); (10) McCook \& Sion (1999); (11) Luyten (1957); (12) Rucinski (2009); (13) Voûte (1918); (14) van Albada (1961); (15) Holberg et al. (1993); (16) Berger \& Fringant (1978); (17) Giclas et al. (1967); (18) Greenstein \& Sargent (1974); (19) Berger \& Fringant (1977); (20) Giclas et al. (1965); (21) Humason \& Zwicky (1947); (22) Greenstein (1966); (23) Luyten (1949); (24) Feige (1958); (25) Baschek \& Sargent (1976); (26) Bonifacio et al. (1995); (27) Pickering \& Cannon (1897); (28) Brown \& Verschueren (1997); (29) Drilling \& Bergeron (1995); (30) this work; (31) Stephenson et al. (1968); (32) Schwartz (1972); (33) Kapteyn (1914); (34) Carnochan \& Wilson (1983); (35) Edelmann et al. (2001); (36) Baschek \& Norris (1970); (37) Gould et al. (1957); (38) Conlon et al. (1990); (39) Martin (2006).

Table 2. New bright blue high proper motion objects.

\begin{tabular}{lccccc}
\hline \hline Albus & 4 & 5 & 6 & 7 & 8 \\
\hline TYC & $6017-419-1$ & $4406-285-1$ & $9044-1653-1$ & $9327-1311-1$ & $4000-216-1$ \\
$\alpha^{a}(\mathrm{~J} 2000)$ & 085158.89 & 142127.86 & 160011.81 & 215341.26 & 233452.08 \\
$\delta^{a}(\mathrm{~J} 2000)$ & -171238.8 & +712421.3 & -643330.3 & -700431.5 & +534702.5 \\
$\mu_{\alpha} \cos \delta\left[\mathrm{mas} \mathrm{a}^{-1}\right]$ & $+0 \pm 2$ & $-62 \pm 2$ & $+3 \pm 3$ & $+10 \pm 2$ & $+68 \pm 2$ \\
$\mu_{\delta}\left[\mathrm{mas} \mathrm{a}{ }^{-1}\right]$ & $-124 \pm 2$ & $-15 \pm 2$ & $-78 \pm 3$ & $-50 \pm 2$ & $+6 \pm 2$ \\
$H_{V_{\mathrm{T}}}[\mathrm{mag}]$ & $11.86 \pm 0.11$ & $10.35 \pm 0.12$ & $11.3 \pm 0.2$ & $10.07 \pm 0.16$ & $10.67 \pm 0.12$ \\
Tycho $B_{\mathrm{T}}[\mathrm{mag}]$ & $11.56 \pm 0.07$ & $10.97 \pm 0.04$ & $11.58 \pm 0.07$ & $11.28 \pm 0.05$ & $11.53 \pm 0.06$ \\
Tycho $V_{\mathrm{T}}[\mathrm{mag}]$ & $11.40 \pm 0.09$ & $11.34 \pm 0.08$ & $11.86 \pm 0.15$ & $11.53 \pm 0.09$ & $11.49 \pm 0.09$ \\
DENIS $i[\mathrm{mag}]$ & $12.06 \pm 0.03$ & $\ldots$ & $12.23 \pm 0.04$ & $11.83 \pm 0.06$ & $\ldots$ \\
2MASS $J[\mathrm{mag}]$ & $12.41 \pm 0.02$ & $11.85 \pm 0.02$ & $12.57 \pm 0.03$ & $12.15 \pm 0.02$ & $12.50 \pm 0.03$ \\
2MASS $H[\mathrm{mag}]$ & $12.55 \pm 0.02$ & $11.95 \pm 0.02^{b}$ & $12.73 \pm 0.04$ & $12.26 \pm 0.03$ & $12.66 \pm 0.03$ \\
2MASS $K_{\mathrm{s}}[\mathrm{mag}]$ & $12.62 \pm 0.03$ & $12.02 \pm 0.03$ & $12.77 \pm 0.04$ & $12.38 \pm 0.03$ & $12.73 \pm 0.02$ \\
GRIZ $g[\mathrm{mag}]$ & 11.51 & 11.17 & $\ldots$ & 11.48 & 11.57 \\
GRIZ $r[\mathrm{mag}]$ & 12.03 & 11.64 & $\ldots$ & 11.95 & 12.13 \\
GRIZ $i[\mathrm{mag}]$ & 12.37 & 11.94 & $\ldots$ & 12.26 & 12.47 \\
GRIZ $z[\mathrm{mag}]$ & 12.67 & 12.21 & $\ldots$ & 12.52 & 12.82 \\
$T_{\text {eff }}^{c}[\mathrm{~K}]$ & 27000 & 24000 & 29000 & 24000 & 30000 \\
\hline
\end{tabular}

Notes. ${ }^{(a)}$ Equatorial coordinates from Tycho-2. ${ }^{(b)}$ Poor $H$-band 2MASS photometry quality (flag “E”). ${ }^{(c)}$ Effective temperatures from the SED fits. 


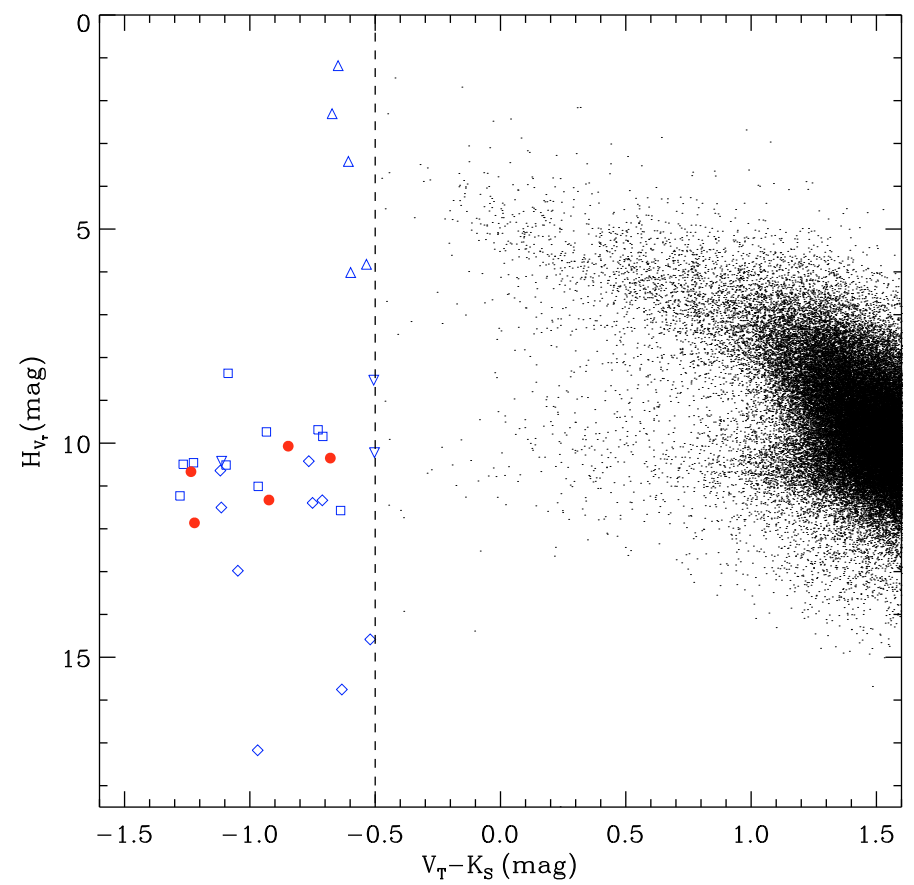

Fig. 2. Reduced proper motion diagram $\left(H_{V_{\mathrm{T}}}\right.$ versus $\left.V_{\mathrm{T}}-K_{\mathrm{S}}\right)$. Selected objects are bluewards of the dashed vertical line at $V_{\mathrm{T}}-K_{\mathrm{s}}=-0.5 \mathrm{mag}$. Previously unreported blue high proper motion objects are depicted with (red) filled circles. Already known blue high proper motion objects are shown with (blue) open symbols: hot subdwarfs with squares, white dwarfs with diamonds, young main-sequence stars with uptriangles, and other stars with down-triangles. Objects redder than $V_{\mathrm{T}}-K_{\mathrm{s}}=-0.5 \mathrm{mag}$ are marked with (black) small dots.

galaxy 2MASX 13052358-4740500 $\quad(z=0.01731 \pm 0.00015$; Crook et al. 2007).

Of the 32 remaining objects, 27 were already known. We list their basic properties in Table 1. Among them are most of the known brightest white dwarfs and hot subdwarfs. Besides, there are runaway OB stars, early-type stars in young moving groups, and one chemically-peculiar halo blue horizontal branch star. Several of them have their trigonometric parallaxes measured by the Hipparcos satellite (van Leeuwen 2007). The trigonometric parallaxes of white dwarfs are larger than those of hot subdwarfs, which indicates longer heliocentric distances and higher luminosities for the latter, consistent with their classification.

The remaining five objects, listed in Table 2, have never before been reported in the literature. For naming them, we followed the "Albus" nomenclature introduced by Caballero \& Solano (2007) and followed in Caballero (2009). Our new Albus objects go from the fourth to the eighth of this series.

\section{Discussion}

The five new bright blue high proper motion objects (Table 2) are placed in the reduced proper motion-colour diagram (Fig. 2) in the region at which hot subdwarfs and early-type white dwarfs gather. To shed some light into the nature of the new Albus objects, we searched for additional photometric data with the "allVO" utility of Aladin. This utility allows the user to query a large number of photometric catalogues in a comfortable way. Besides Tycho-2 and 2MASS, two others catalogues had additional photometric data: DENIS and GRIZ (Ofek 2008). DENIS gives magnitudes in the $i$ band for the three Albus sources in the Southern hemisphere, whereas the GRIZ catalogue provides

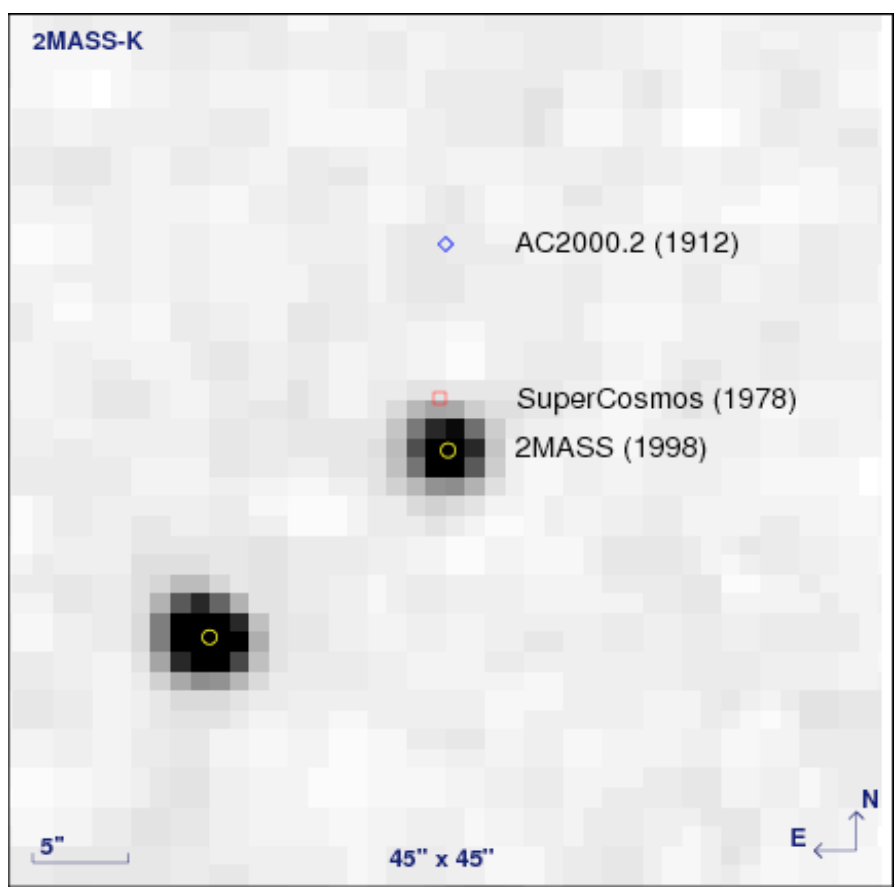

Fig. 3. Verification of the high proper motion of Albus 4 (TYC 6017419-1), a representative blue high proper motion object, in a $K_{\mathrm{s}}$-band 2MASS image. The epochs of observation in the AC2000.2 (blue diamond), SuperCOSMOS (red square), and 2MASS (yellow circles) are shown in parenthesis. The DENIS epoch almost coincides with the 2MASS one.

synthetic SDSS griz magnitudes for all new Albus sources but Albus 6 (see Table 2). The typical uncertainty for the GRIZ photometry is about $0.12,0.12,0.10$, and $0.08 \mathrm{mag}(1 \sigma)$, for the $g$, $r, i$, and $z$-bands, respectively.

We took advantage of another VO-tool, VOSA ${ }^{4}$ (VO Sed Analyzer) to fit the observed spectral energy distributions (SEDs) to theoretical models. The VOSA allows the user to query in an automatic and transparent way different collections of theoretical models, to calculate their synthetic photometry, and to perform a statistical test to determine which model best reproduces the observed data. To fit the SEDs of our Albus objects, we used only the observational data (all but the GRIZ ones) and fitted them to Kurucz stellar atmosphere models (Castelli et al. 1997). The effective temperatures $T_{\text {eff }}$ obtained with VOSA ranged between 24000 and $30000 \mathrm{~K}$ (see Table 2). The accuracy of the effective temperature estimated with this method is defined by the step of the grid of the model, $1000 \mathrm{~K}$ in this case. In Fig. 4 we have plotted the theoretical fitting of the observational SEDs.

The confirmation of the real nature of these objects requires spectroscopic data. To look for spectra in the VO archives, we used another VO-tool, VOSED ${ }^{5}$. Developed by the Spanish Virtual Observatory, VOSED allows the user to gather spectroscopic information available throughout the VO. Only Albus 5 was found to have spectroscopic data. In particular, it was observed by the Far Ultraviolet Spectroscopic Explorer (FUSE) as part of the programme G061. We used this spectrum to confirm its hot subdwarf nature. In Fig. 5 we compare the FUSE spectra of Albus 5 to the well known sdB star HD 205805 ( $T_{\mathrm{eff}} \gtrsim 26000 \mathrm{~K}$; Baschek \& Norris 1970). The two spectra are

\footnotetext{
4 http://svo.cab.inta-csic.es/theory/vosa

5 http://sdc.cab.inta-csic.es/vosed
} 
F. M. Jiménez-Esteban et al.: Blue high proper motion objects in Tycho-2 and 2MASS

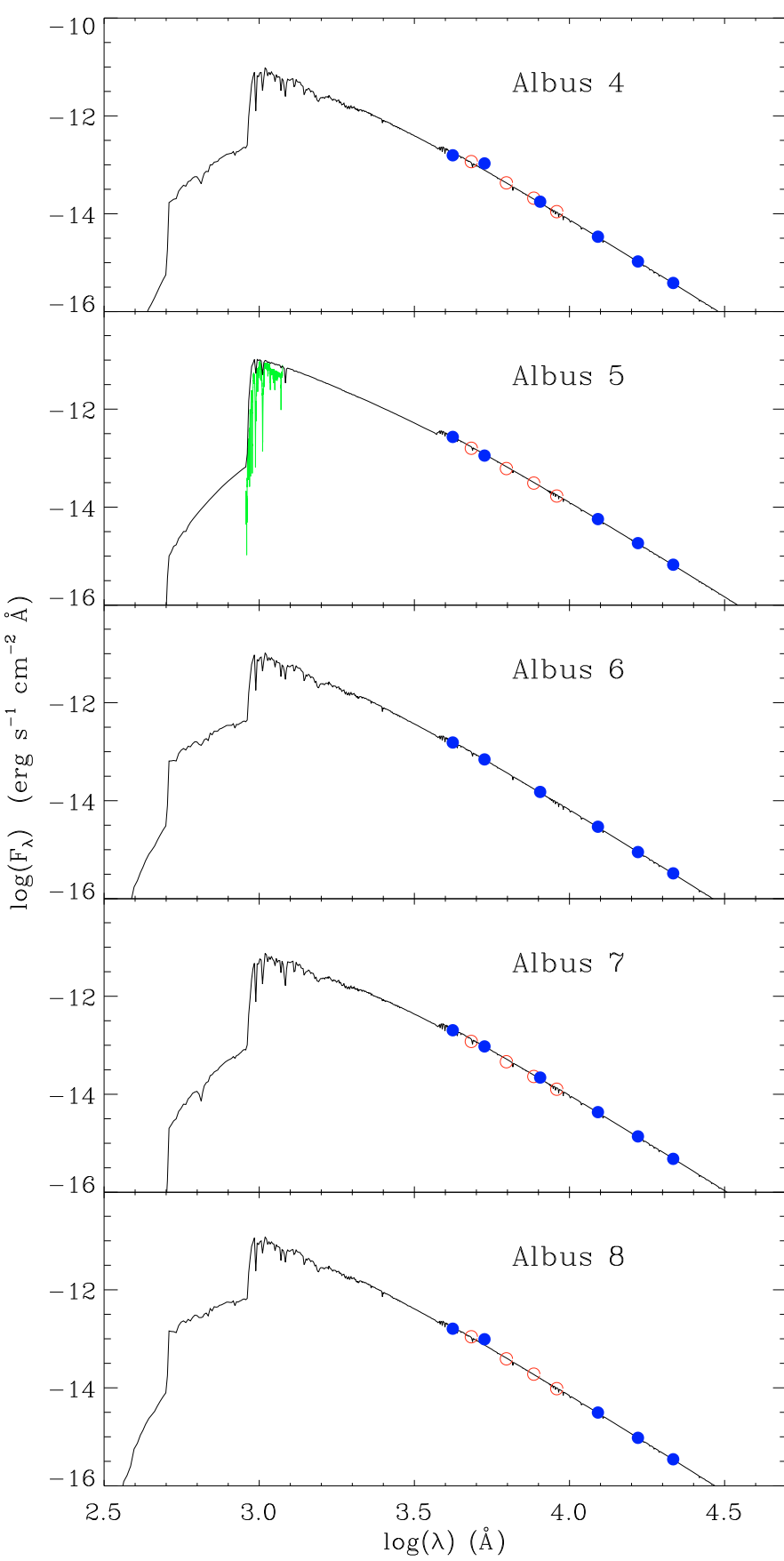

Fig. 4. Theoretical fitting of the Albus SEDs. Solid lines represent the Kurucz models that best fits the observational data. Filled (blue) and open (red) circles indicate the photometric data used for the fit and the synthetic GRIZ photometry data used for control, respectively. The thin (green) line in the second panel depicts the ultraviolet FUSE spectra of Albus 5 .

almost identical, showing a huge number of photospheric and interstellar absorption lines that are observed in the FUSE spectra of almost all sdB stars (Chayer et al. 2006). Because of the strong similitude of these two ultraviolet spectra and the $T_{\text {eff }}$ of $24000 \mathrm{~K}$ obtained using VOSA, we classify Albus 5 as a bonafide sdB star. The other four objects have equal or higher $T_{\text {eff }}$ (and bluer $V_{\mathrm{T}}-K_{\mathrm{s}}$ colours) and could be either sdO/B subdwarfs or early-type white dwarfs. Knowing their trigonometric parallaxes would help to classify them. Unfortunately, neither of the five new Albus are included in the Hipparcos catalogue.

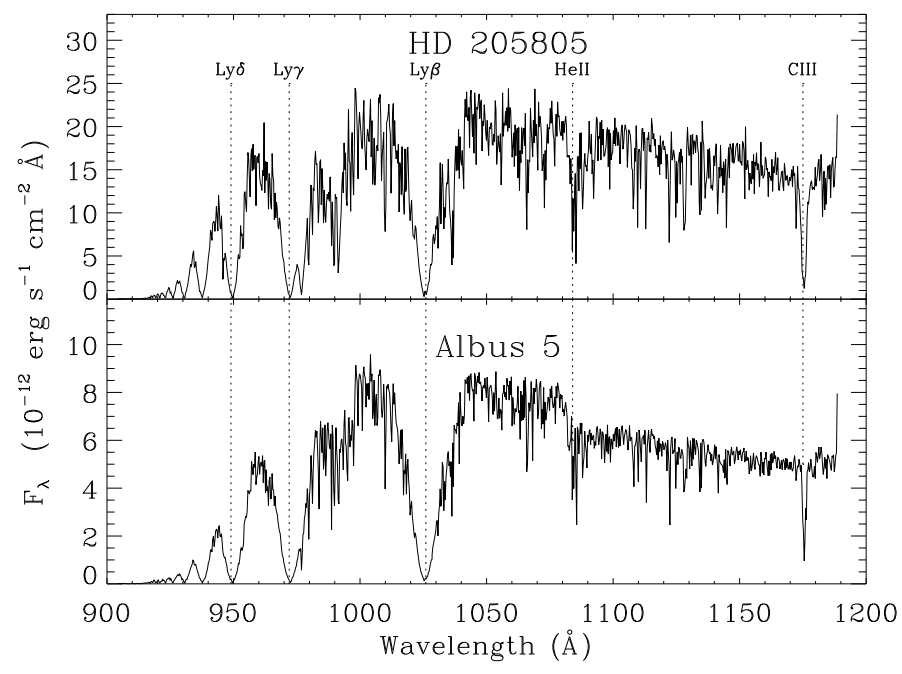

Fig. 5. FUSE spectra of the sdB star HD 205805 (upper panel) and Albus 5 (lower panel). The strongest observed lines (e.g. of the Lyman series) and multiplets (e.g. C III) have been labelled.

\section{Summary}

To find out blue high proper motion objects, we cross-matched the 2.5 million sources in the Tycho-2 catalogue with the 471 million sources in the 2MASS catalogue. From this correlation, and after discarding one erroneous identification, we obtained a sample of 32 objects with proper motions larger than $50 \mathrm{mas} \mathrm{yr}^{-1}$ and $V_{\mathrm{T}}-K_{\mathrm{S}}$ colours bluer than $-0.5 \mathrm{mag}$. Of these, 27 were known and five (Albus 4 to 8 ) were studied here for the first time.

From the position of the new discovered sources in a reduced proper motion-colour diagram and theoretical fitting of their SEDs, we concluded that these objects are probably hot subdwarfs or early-type white dwarfs. For Albus 5, we confirmed its nature as an sdB star with a FUSE far-ultraviolet spectrum. For the remaining objects, spectroscopic follow-up observations are required to ascertain their true nature. In any case, because of their relative brightness and SED profiles, the five objects can serve as useful spectro-photometric standards or be the subject of dedicated studies (e.g., pulsations and origin of hot subdwarfs, metallic abundances and atmospheric parameters in white dwarfs).

Acknowledgements. This work was partially funded by the Spanish Ministerio de Ciencia e Innovación under the Consolider-Ingenio 2010 Program grant CSD2006-00070 First Science with the Gran Telescopio Canarias. This research has made use of VOSA and VOSED, developed by the Spanish Virtual Observatory through grants AyA2008-02156 and RI031675, and of Aladin and SIMBAD developed at the Centre de Données astronomiques de Strasbourg, France. Financial support was provided by the Universidad Complutense de Madrid, the Comunidad Autónoma de Madrid, and the Spanish Ministerio de Ciencia e Innovación under grants AyA2008-00695, AyA2008-06423-C03-03, SP2009/ESP-1496.

\section{References}

Argelander, F. 1903, Bonner Durchmusterung des nordlichen Himmels (Bonn:

Marcus and Weber's Verlag)

Baschek, B., \& Norris, J. 1970, ApJS, 19, 327

Baschek, B., \& Sargent, A. I. 1976, A\&A, 53, 47

Berger, J., \& Fringant, A. 1977, A\&AS, 28, 123

Berger, J., \& Fringant, A. 1978, A\&A, 64, L9

Bonifacio, P., Castelli, F., \& Hack, M. 1995, A\&AS, 110, 441

Bonnarel, F., Fernique, P., Bienaymé, O., et al. 2000, A\&AS, 143, 33

Brown, A. G. A., \& Verschueren, W. 1997, A\&A, 319, 811 
Caballero, J. A. 2009, Multi-wavelength Astronomy and Virtual Observatory, Proceedings of the EURO-VO Workshop, held at the European Space Astronomy Centre of ESA, Villafranca del Castillo, Spain, 1-3 December, 2008, ed. D. Baines, \& P. Osuna, published by the European Space Agency, 3

Caballero, J. A., \& Solano, E. 2007, ApJ, 665, L151

Cannon, A. J., \& Pickering, E. 1925, The Henry Draper (HD) Catalogue, HD extension, ed. A. J. Cannon, \& E. Pickering, 100

Carnochan, D. J., \& Wilson, R. 1983, MNRAS, 202, 317

Castelli, F., Gratton, R. G., \& Kurucz, R. L. 1997, A\&A, 318, 841

Chayer, P., Oliveira, C., Dupuis, J., Moos, H. W., \& Welsh, B. Y. 2006, in Astrophysics in the Far Ultraviolet: Five Years of Discovery with FUSE, ed. G. Sonneborn, H. W. Moos, \& B.-G. Andersson, ASP Conf. Ser., 348, 209

Conlon, E. S., Dufton, P. L., Keenan, F. P., \& Leonard, P. J. T. 1990, A\&A, 236, 357

Crook, A. C., Huchra, J. P., Martimbeau, N., et al. 2007, ApJ, 655, 790

Drilling, J. S., \& Bergeron, L. E. 1995, PASP, 107, 846

Edelmann, H., Heber, U., \& Napiwotzki, R. 2001, AN, 322, 401

Epchtein, N., de Batz, B., Capoani, L., et al. 1997, The Messenger, 87, 27

Feige, J. 1958, ApJ, 128, 267

Giclas, H. L., Burnham, R., \& Thomas, N. G. 1965, Lowell Observatory Bulletin, 6,155

Giclas, H. L., Burnham, R., \& Thomas, N. G. 1967, Lowell Observatory Bulletin, 7,49

Gould, N. L., Herbig, G. H., \& Morgan, W. W. 1957, PASP, 69, 242

Greenstein, J. L. 1966, ApJ, 144, 496

Greenstein, J. L. 1969, ApJ, 158, 281

Greenstein, J. L., \& Sargent, A. I. 1974, ApJS, 28, 157

Hambly, N. C., MacGillivray, H. T., Read, M. A., et al. 2001, MNRAS, 326, 1279

Haro, G., \& Luyten, W. J. 1962, Boletin de los Observatorios Tonantzintla y Tacubaya, 3, 37

Høg, E., Fabricius, C., Makarov, V. V., et al. 2000, A\&A, 355, L27

Holberg, J. B., Barstow, M. A., Buckley, D. A. H., et al. 1993, ApJ, 416, 806

Hoogerwerf, R., de Bruijne, J. H. J., \& de Zeeuw, P. T. 2001, A\&A, 365, 49

Humason, M. L., \& Zwicky, F. 1947, ApJ, 105, 85

Kapteyn, J. C. 1914, ApJ, 40, 43
Kilkenny, D., Heber, U., \& Drilling, J. S. 1988, South African Astronomical Observatory Circular, 12, 1

Lépine, S., \& Shara, M. M. 2005, AJ, 129, 1483

Luyten, W. J. 1949, ApJ, 109, 528

Luyten, W. J. 1957, in The Observatory, Univ. Minnesota, Minneapolis, 1953, 9,1

Martin, J. C. 2006, AJ, 131, 3047

McCook, G. P., \& Sion, E. M. 1999, ApJS, 121, 1

Monet, D. G., Levine, S. E., Canzian, B., et al. 2003, AJ, 125, 984

Ofek, E. O. 2008, PASP, 120, 1128

Oke, J. B. 1990, AJ, 99, 1621

Oke, J. B., \& Gunn, J. E. 1983, ApJ, 266, 713

O'Toole, S. J., \& Heber, U. 2006, A\&A, 452, 579

Perryman, M. A. C., Lindegren, L., Kovalevsky, J., et al. 1997, A\&A, 323, L49

Pickering, E. C., \& Cannon, A. J. 1897, ApJ, 6, 349

Röser, S., Schilbach, E., Schwan, H., et al. 2008, A\&A, 488, 401

Rucinski, S. M. 2009, MNRAS, 395, 2299

Schonfeld, E. 1886, in Bonner Durchmusterung des nordlichen Himmels (Bonn: Marcus and Weber's Verlag)

Schwartz, R. D. 1972, PASP, 84, 28

Sion, E. M., Holberg, J. B., Oswalt, T. D., McCook, G. P., \& Wasatonic, R. 2009, AJ, 138, 1681

Skrutskie, M. F., Cutri, R. M., Stiening, R., et al. 2006, AJ, 131, 1163

Stephenson, C. B., Sanduleak, N., \& Hoffleit, D. 1968, PASP, 80, 92

Stone, R. P. S. 1977, ApJ, 218, 767

Thackeray, A. D. 1961, Monthly Notes of the Astronomical Society of South Africa, 20, 40

Thome, J. M. 1894, Cordoba Durchmusterung. Brightness and position of every fixed star down to the 10 . magnitude comprised in the belt of the heavens between 32 and 90 degrees of southern declination $-32 \mathrm{deg}$. to $-42 \mathrm{deg}$., ed. J. M. Thome, 17

Urban, S. E., Corbin, T. E., Wycoff, G. L., et al. 1998, AJ, 115, 1212

van Albada, T. S. 1961, Bull. Astron. Inst. Netherlands, 15, 301

van Leeuwen, F. 2007, A\&A, 474, 653

Voûte, J. 1918, ApJ, 48, 144

Zuckerman, B., Song, I., \& Webb, R. A. 2001, ApJ, 559, 388 